\title{
COMPARAÇÃO DA ATIVIDADE INSETICIDA DE Chenopodiumambrosoides E Azadirachta indica NO CONTROLE DE Sitophilus zeamais
}

\author{
Edvan Costa da Silva ${ }^{1}$, Deilton Damas Vieira ${ }^{2}$, Léo Vieira Leonel ${ }^{3}$ \\ ${ }^{1}$ Pós-graduando (Mestrado) Produção Vegetal, Universidade Estadual do Goiás / UEG, Ipameri - GO. \\ edvan_costa@outlook.com \\ ${ }^{2}$ Engenheiro Agrônomo, Universidade Estadual do Maranhão / UEMA, Imperatriz - MA. \\ ${ }^{3}$ Mestre em Agroecologia, Universidade Estadual do Maranhão / UEMA, São Luís - MA.
}

RESUMO: Este trabalho teve como objetivo avaliar a atividade inseticida de Chenopodium ambrosoides (Mastruz) e de Azadirachta indica (Nim), no controle de Sitophilus zeamais. Foi avaliada a mortalidade em teste sem chance de escolha, e repelência em teste com chance de escolha. No primeiro teste avaliou-se o efeito inseticida dos pós sobre a fase adulta do inseto, utilizando-se potes plásticos contendo $10 \mathrm{~g}$ de milho. No segundo teste avaliou-se a repelência utilizando-se arenas formadas por cinco caixas plásticas circulares com tampas. $C$. ambrosoides causou mortalidade de $100 \%$ nos tratamentos com 0,3 e $0,5 \mathrm{~g}$ do pó. A. indica alcançou uma taxa de mortalidade de $89 \%$ na maior concentração do pó. Foi comprovado efeito repelente para as duas espécies utilizadas.

Palavras-chave: Gorgulho-do-milho. Inseticidas naturais. Mastruz. Nim.

\section{COMPARISON OF THE INSECTICIDAL ACTIVITY OF Chenopodiumambrosoides AND Azadirachtaindica IN THE CONTROL OF Sitophilus zeamais}

\begin{abstract}
This study aimed to evaluate the insecticide Chenopodium ambrosoides activity (Mastruz) and Azadirachta indica (Neem), for the control of Sitophilus zeamais. It was evaluated mortality in a no choice test, and repellency in a free choice test. In the first test evaluated the insecticidal effect on adult insect, using plastic pots containing $10 \mathrm{~g}$ of corn. In the second test evaluated the repellency using arenas formed with five circular plastic boxes. C. ambrosoides caused $100 \%$ mortality on treatment with 0.3 and $0.5 \mathrm{~g}$ of powder. The A. indica has caused $89 \%$ mortality rate at the highest powder concentration. It was proven repellent effect for the two species used.
\end{abstract}

Key words: Maize Weevil. Mastruz. Natural insecticides. Nem.

\section{INTRODUÇÃO}

O milho, Zeamays L. (Poaceae), é cultivado na maioria dos países, representando o segundo cereal mais importante em termos de produção mundial, suplantado apenas pelo trigo. No Brasil, as perdas devido ao ataque de pragas chegam à $20 \%$ da produção total do grão, demandando a necessidade de novas pesquisas e tecnologias (SILVA et al., 2007).

Cultura Agronômica, Ilha Solteira, v.26, n.4, p.554-559, 2017 
O gorgulho-do-milho, SitophiluszeamaysMots. (Coleoptera: Curculionidae) destaca-se como uma das principais pragas do milho e de outros cereais armazenados, no Brasil, provocando perdas de peso dos grãos, desvalorização comercial, perda no valor nutritivo e diminuição no poder germinativo das sementes. $O$ inseto possui grande número de hospedeiros, elevado potencial biótico, capacidade de penetração na massa de grãos e possibilidade de infestação tanto no campo como nas unidades de armazenamento (LORINI, 2008).

Diversas pesquisas têm demonstrado a viabilidade do uso de compostos bioativos obtidos de plantas no controle de pragas de grãos armazenados, devido a sua eficiência, geralmente de baixo custo, segurança para os aplicadores, consumidores e meio ambiente (DEMISSIE et al., 2008).

O emprego de inseticidas botânicos no controle de pragas de grãos armazenados mostrase bastante promissor principalmente tendo em vista a possibilidade de se controlarem as condições ambientais no interior das instalações de armazenamento, propiciando a maximização da atividade inseticida. Nesses locais, os produtos podem ser empregados na forma de pós, extratos e óleos (TAVARES; VENDRAMIM, 2005).

A Azadirachta indica A. Juss, conhecida comumente por nim é utilizado há séculos nos países do oriente. Na Índia seu país de origem, seu uso se dá de diversas formas: planta medicinal, combustível, planta sombreadora, adubo, lubrificante, e nas últimas décadas seu estudo tem se difundido devido às substâncias inseticidas presentes nas folhas e frutos. (MOSSINI; KEMMELMEIER, 2005) e C. ambrosioides foi estudado e verificado sua eficiência empulgão preto (Toxopteracitricida) em citros por Tavares eVendramim, (2005).

Assim esse trabalho teve como objetivo avaliar o efeito do pó de C.ambrosoides (Mastruz) e A. indica (Nim) em diferentes concentrações na mortalidade em teste sem chance de escolha; e repelência em teste com chance de escolha, no controle de adultos de S.zeamaisM.

\section{MATERIAL E MÉTODOS}

Os experimentos Foram realizados no laboratório de Entomologia da Universidade Estadual do Maranhão CESI-UEMA, no período de Março de 2014 a Agosto de 2014. Os gorgulhos foram coletados em visitas a pequenos agricultores que não utilizam inseticidas, sendo que os insetos com idade de até 10 dias foram acondicionados em uma arena contendo cinco caixas plásticas circulares $(6,1 \mathrm{~cm}$ de diâmetro e $2,1 \mathrm{~cm}$ de altura), sendo uma central interligada às outras por cilindros plásticos.

C. ambrosioidesL.foi coletado no povoado de Sete Barracas, Município de São Miguel do Tocantins e A. indicafoi obtida na feira do produtor em São Miguel do Tocantins - TO.

Obtenção do extrato em pó

Cultura Agronômica, Ilha Solteira, v.26, n.4, p.554-559, 2017 
As folhas de A. indicae a planta inteira de C.ambrosioides foram coletadas e secadas em estufa com ventilação forçada de ar a $40^{\circ} \mathrm{C}$, durante 48 horas dentro de sacos de papel. Depois de secas, foramtrituradas em um liquidificador até obtenção de um fino pó, que foi armazenado em recipientes de vidro hermeticamente fechados, em ambiente protegido ao abrigo de luz e umidade até sua utilização.

Avaliação da mortalidade dos adultos em teste sem chance de escolha

Para o experimento sem chance de escolha foram utilizadas caixas plásticas circulares $(6,1 \times 2,1 \mathrm{~cm})$ e transparentes, contendo 10 gramas de grãos de milho com dosagens de $0.1,0.3,0.5 \mathrm{~g}$ do pó de $A$. indica e C.ambrosioides,respectivamente, a fim de se determinar a dosagem mínima para controle de $100 \%$ dos adultos de $S$. zeamais,onde foram colocados 10 gorgulhos, em cada recipiente. Foram realizados sete tratamentos com oito repetições cada, incluindo a testemunha. Para a avaliação da mortalidade, foram contabilizados os números de insetos mortos durante cinco dias.

\section{Avaliação da repelência dos adultos em teste com chance de escolha}

O experimento com chance de escolha foi realizado testando-se o pó de uma planta por vez, em arenas formadas por cinco caixas plásticas circulares com tampas, sendo uma caixa central interligada simetricamente a outras quatro por tubos plásticos com distância de $10 \mathrm{~cm}$, em diagonal seguindo a metodologia utilizada por Tavares e Vendramim (2005). As quatro caixas das diagonais receberam $10 \mathrm{~g}$ de grãos de milho, sendo nos potesA, B e C tratadas com o pó de uma das plantas contendo $0.5 \mathrm{~g}$ e o pote $\mathrm{D}$ contendo apenas o milho (testemunha). $\mathrm{Na}$ caixa central, pote E, foram colocados 10 gorgulhos e, após 24 h, foi contado o número de insetos por recipiente.

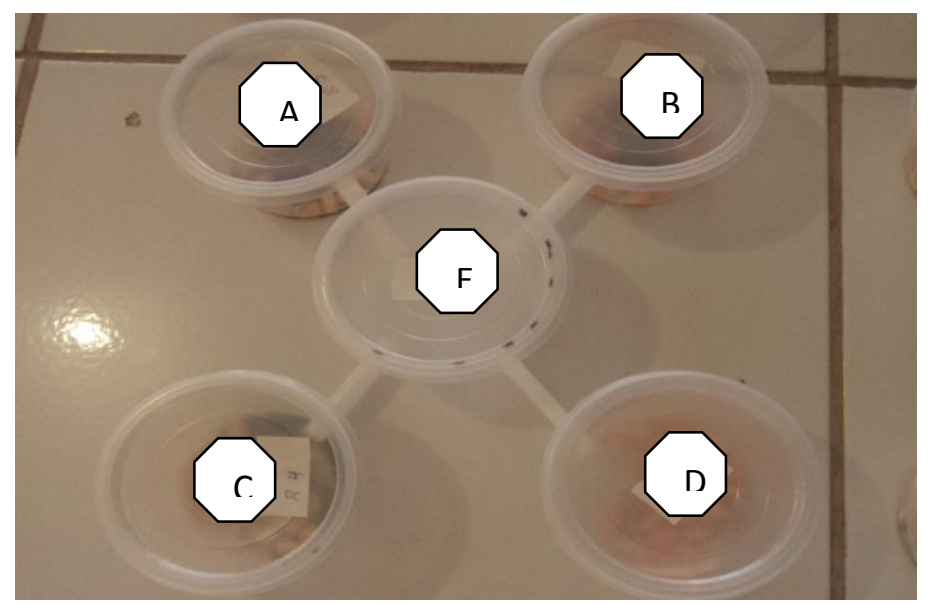

Figura 1. Arena utilizada para avaliação da repelência dos diferentes pós de origem vegetal sobre S. zeamais.

O delineamento foi inteiramente casualizado, com 2 tratamentos (um tratamento para A. indica e outro para $C$. ambrosioides constituídos de 4 repetições incluindo a testemunha. As análises de variância dos experimentos foram feitas através do teste $\mathrm{F}$ e os resultados 
obtidos comparados através do teste de Tukey a 5\% de probabilidade. Os resultados foram processados pelo software ASSISTAT 7.7 beta.

\section{RESULTADOS E DISCUSSÃO}

Pôde-se observar que no teste de mortalidade de adultos, sem chance de escolha, entre as duas espécies vegetais testadas, $C$. ambrosioides nas concentrações $0,3 \mathrm{~g}$ e $0,5 \mathrm{~g}$ de pó/ 10 g de grãos foi altamente tóxico aos adultos de $S$. zeamais, causando $100 \%$ de mortalidade após 5 dias, sendo então diferente estatisticamente pelo teste de Tukey ao nível de 5\% de probabilidade (Tabela 1).

Tabela 1. Porcentagem média de mortalidade de adultos de $S$. zeamais em grãos de milho tratados com pós de vegetais, em um período de 5 dias de avaliação. Teste sem chance de escolha.

\section{Tratamentos}

Chenopodiumambrosioides $(0,5 \mathrm{~g})$

Chenopodiumambrosioides $(0,3 \mathrm{~g})$

Azadirachta indica $(0,5 \mathrm{~g})$

Chenopodiumambrosioides $(0,1 \mathrm{~g})$

Azadirachta indica $(0,3 \mathrm{~g})$

Azadirachta indica $(0,1 \mathrm{~g})$

Testemunha

\section{Mortalidade - \% ( \pm Desvio Padrão)}

$$
\begin{gathered}
100,0( \pm 0,00) \mathrm{a} \\
100,0( \pm 0,00) \mathrm{a} \\
89,0( \pm 1,13) \mathrm{a} \\
54,0( \pm 3,25) \mathrm{b} \\
47,0( \pm 3,84) \mathrm{b} \\
32,0( \pm 2,43) \mathrm{bc} \\
20,0( \pm 1,41) \mathrm{c}
\end{gathered}
$$

As médias seguidas pela mesma letra não diferem estatisticamente entre si pelo Teste de Tukey ao nível de 5\% de probabilidade, encontrados os valores de F (17.582) e P $(<0005)$.

E com a redução da concentração ( $0,1 \mathrm{~g}$ de pó / $10 \mathrm{~g}$ de grãos) deC. ambrosioides, observou-se que a mortalidade era reduzida, então nesta concentração obteve-se uma mortalidade de $54 \%$, sendo um valor não significativo estatisticamente se comparados com as concentrações de0,3g e 0,5g de pó/ $10 \mathrm{~g}$ de grãos da mesma espécie em estudo.

Resultado semelhante foi encontrado por Tavares e Vendramim (2005) testando o efeito inseticida de $C$. ambrosioides sobre $S$. zeamais. Procópio et al. (2003), trabalhando com pós de origemvegetal, encontraram total mortalidade de adultos de $S$. zeamais quando expostos a grãos de milho tratados coma mistura de pós de folhas, flores e frutos de $C$. ambrosioides. Alta mortalidade de adultos dessa pragatambém foi obtida com a mistura de grãos de milho compós de C. ambrosioides (SILVA et al., 2003).

No teste realizado com A. indica, foi observado $89 \%$ da mortalidade no tratamento com a concentração de $0,5 \mathrm{~g}$ de pó/ $10 \mathrm{~g}$ de grãos, tendo valor significativo estatisticamente. As demais concentrações ficaram abaixo de $60 \%$ de mortalidade, sendo a mortalidade de $47 \%$ e $32 \%$ nas concentrações $0,3 \mathrm{~g}$ de pó/ 10 de grãos e $0,1 \mathrm{~g}$ de pó/ 10 grãos, respectivamente, não tendo valores significativos estatisticamente ao Teste de Tukey ao nível de 5\% de probabilidade.Para a testemunha, os valores não diferiram estatisticamente, já que não houve mortalidade de insetos. 
$\mathrm{Na}$ avaliação da repelência dos adultos em teste com chance de escolha, foi aplicado o teste $t$ de Student ao nível de 5\% de probabilidade. Ao comparar a testemunha com as espécies $A$. indica e $S$. zeamais, em ambos os casos houve diferença estatística entre as médias avaliadas, corroborando para os efeitos positivos das plantas na redução das populações de insetos pragas de grãos armazenados. Quando comparado à espécie A. indica com S. zeamais, não deferiu estatisticamente (p-valor superior a $0,05 \%$ ), pois ambas as espécies contribuíram positivamente para a diminuição dos insetos nas amostras de milho.

Os insetos repelidos pelo milho tratado com pó das plantas testadas foram superiores aos das testemunhas, deixando claro que as espécies $A$. indicae $S$. zeamais estudadas na presente pesquisa mostraramefeitos significativos de repelência sobre os insetos (Tabela 2).

Tabela 2. Repelência de adultos de $S$. zeamais por pós vegetais. Teste com chance de escolha.

\begin{tabular}{lc}
\hline \multicolumn{1}{c}{ Tratamentos } & Adultos atraídos - \% ( \pm Desvio Padrão $)$ \\
\hline Azadirachta indica & $60,0( \pm 6,44) \mathrm{a}$ \\
Testemunha & $30,00( \pm 3,30) \mathrm{b}$ \\
\hline Chenopodiumambrosioides & $52,5( \pm 5,26) \mathrm{a}$ \\
Testemunha & $40,00( \pm 2,73) \mathrm{b}$ \\
\hline
\end{tabular}

As médias seguidas pela mesma letra não diferem estatisticamente entre si. Foi aplicado o Teste de $t$ student com duas amostras presumindo variâncias diferentes ao nível de $5 \%$ de probabilidade. Sendo o valor de $\mathrm{P}(\mathrm{T}<=\mathrm{t})$ bi-caudal 0,4192 e t crítico bi-caudal 3,1824.

Resultados que corroboram com Silva et al. (2007), testando o pó de diferentes espécies vegetais em grão de milho armazenado, relataram $A$. indica como eficiente no controle do $S$. zeamais.

De acordo com Tavares e Vendramim (2005), não foi encontro efeito repelente e nem atrativo dos pós das folhas e frutos de C.ambrosioides em relação aos adultos de $S$. zeamais, esse resultado difere do que foi citado por Silva et al. (2005), que observaram efeito repelente dessa planta sobre $S$. zeamais.

Mendonça et al. (2013), também constataram efeito repelente de pós de Cymbopogonsp (citronela), C. ambrosioides (mastruz) e C. citratus (capim-santo) e efeito inseticida de Chenopodiumambrosioides L. (mastruz) sobre Sitophilus sp. Isso indica que outras espécies vegetais podem ser constituídas de substâncias que causem a repelência a esse tipo de inseto.

\section{CONCLUSÃO}

O uso de C. ambrosioides (mastruz) nas dosagens 0,3 e $0,5 \mathrm{~g} / 10 \mathrm{~g}$ de milho, causou $100 \%$ de mortalidade, provando seu efeito tóxico aos adultos de $S$. zeamais.

O tratamento realizado com A. indica (Nim), na dosagem $0,5 \mathrm{~g} / 10 \mathrm{~g}$ de milho, apresenta taxa de mortalidade próxima aos $90 \%$, podendo também ser recomendada como tóxica aos adultos de $S$. zeamais.

C. ambrosioides e A. indica mostram efeito repelente sobre os adultos de $S$. zeamais.

Cultura Agronômica, Ilha Solteira, v.26, n.4, p.554-559, 2017 
É comprovada a eficiência do pó de $C$. ambrosioides na mortalidade e repelência dos insetos.

\section{REFERÊNCIAS BIBLIOGRÁFICAS}

DEMISSIE, G.; TESHOME, A.; ABAKEMAL, D.; TADESSE, A. Cooking oils and "Triplex" in the control of Sitophilus zeamais Motschulsky (Coleoptera: Curculionidae). JournalofStoredProductsResearch, Amsterdã, v. 44, n. 2, p.173-178, 2008.

LORINI, I. Manejo integrado de pragas de grãos de cereais armazenados. 2. ed. Passo Fundo: Embrapa Trigo, 2008.71 p.

MENDONÇA, A. L.; BROGLIO, S. M. F.; ARAÚJO, A. M. N.; LOPES, D. O. P.; PINI, N. S. D. Efeito de pós vegetais sobre o Sitophilus sp. (Mots.1855) (Coleoptera:Curculionidae). Arquivos do Instituto Biológico, São Paulo, v.80, n.1, p.91-97, 2013.

MOSSINI, S. A. G.; KEMMELMEIER, C. A árvore nim (Azadirachta indica A. Juss): múltiplos usos. Acta Farmacêutica Bonaerense, Buenos Aires, v.24, n.1, p.139-148, 2005.

PROCÓPIO, S. O.; VENDRAMIM, J. D.; JÚNIOR, J. R.; SANTOS, J. B. Bioatividade de diversos pós de origem vegetal em relação A SitophiluszeamaisMots. (Coleoptera: Curculionidae). Ciência eAgrotecnologia, Lavras,v.27, n.6, p.1231-1236, 2003.

SILVA, P. H.; TRIVELIN, P. C. O.; GUIRADO, N.; AMBROSANO, E. J.; MENDES, P. C. D.; ROSSI, F.; ARÉVALO, R. A. Controle alternativo de Sitophiluszeamais(Col.: Curculionidae) em grãos de milho. Revista Brasileira de Agroecologia, Guarapari, v.2, n.1, p.902-905, 2007.

SILVA, G.; ORREGO, R. H.; TAPIA, M. Búsqueda de plantas conpropiedadesinsecticidas para elcontrol de Sitophiluszeamais enmaízalmacenado. Pesquisa Agropecuária Brasileira, Brasília, v. 40, n.1, p.11-17, 2005.

SILVA, G.; LAGUNES, A.; RODRÍGUEZ, J. Control de Sitophiluszeamais (Coleoptera: Curculionidae) com polvos vegetales solos y em mesclas com carbonato de cálcio em maizalmacenado. Ciencia eInvestigación Agraria, Santiago, v.30, n.3, p.153-160. 2003.

TAVARES, M. A. G. C.; VENDRAMIM, J.D. Bioatividade da Erva-de-Santa-Maria, Chenopodiumambrosioides L., Sobre SitophiluszeamaisMots. (Coleoptera: Curculionidae). Neotropical Entomology, Londrina, v.34, n.2, p.319-323, 2005. 